\title{
Microstructural Characterization in Aluminum-Graphite Composites Produced by Mechanical Milling and Hot Extrusion
}

\author{
M. I. Flores -Zamora, I. Estrada-Guel, M. Miki-Yoshida and R. Martínez-Sánchez \\ Centro de Investigación en Materiales Avanzados (CIMAV), Miguel de Cervantes No.120, C.P. \\ 31109, Chihuahua, Chih., México.
}

Aluminum alloys have a great diversity of industrial applications because of their low density and good workability, but the use of these alloys is limited due to their relatively low yield stress. Recently, the interest to increase aluminum strength for applications in the aerospace and aeronautics industries has motivated the study of aluminum matrix composites (AMC) which could present better mechanical properties at both, medium $(473 \mathrm{~K})$ and room temperatures. The raw powder materials used were $\mathrm{Al}$ (99.5\% purity, -325 meshes in size) and graphite nanoparticles. The selected graphite content was $0.0,0.25,0.50,0.75$, and $1.0 \mathrm{wt}$. \%. Each mixture was mechanically milled in a high energy SIMOLOYER mill for $2 \mathrm{hrs}$ in an argon atmosphere.

Consolidated bulk products ( $4 \mathrm{~cm}$ of diameter $\phi)$ were prepared by pressing the milled powder at $\sim 950 \mathrm{MPa}$ for two minutes under uniaxial load. Pressed samples were next sintered under vacuum for $3 \mathrm{hrs}$ at $823 \mathrm{~K}$ with a heating rate of $50 \mathrm{~K} / \mathrm{min}$.

Sintered products were held for $0.5 \mathrm{~h}$ at $823 \mathrm{~K}$ and hot extruded into a rod of $10 \mathrm{~mm}$ diameter by using indirect extrusion and an extrusion ratio of 16. Table I shows the sample identification, composition and experimental conditions used.

Tension and compression tests were done in an INSTRON testing machine at room temperature and at constant displacement rate of $0.016 \mathrm{~mm} / \mathrm{s}$. The yield stress was measured at the elastic limit. The microstructural characterization was done by Transmission Electron Microscopy (TEM) in a Philips CM 200 equipped with EDS and PEELS analyzers.

Figure 1 shows the $\sigma$ vs $\varepsilon$ tension curves of the aluminum graphite microcomposites samples tested in the extrusion direction as a function of graphite content. All samples follow a millingsintering-extrusion sequence, with exception of $\mathrm{Al}_{\mathrm{p}}$ sample, which was only sintered and extruded. From this Figure it is evident the increment of the resistance upholding the ductility in all the samples.

Figures 2 and 3 present $\sigma_{\mathrm{y}}$ and $\sigma_{\max }$ values found in present work. From these Figures it is manifest the important effect of graphite content in mechanical properties; both, $\sigma_{y}$ and $\sigma_{\max }$ present a positive slope as a function of graphite content. $\sigma_{\mathrm{y}}$ variation was from 11.4 to 15.11 $\mathrm{kg} / \mathrm{mm}^{2}$ which correspond to an increment of about $34 \%$, and for $\sigma_{\max }$ increment was around 28 $\%$. This is an important enhancement of the mechanical resistance, considering also that the ductility of the samples was kept, as it is shown in Figure 1.

Figure 4 shows a representative microstructure found in $\mathrm{Al}_{\mathrm{p}}$ sample in the as-extruded condition. In this sample was observed the presence of both, equiaxial and deformed grains, due to partial recrystallization during hot extrusion. Figure 5 shows a representative view of reinforcement nanoparticles, which present sizes close to $50 \mathrm{~nm}$ and their distribution, is at random. Previous analysis showed the presence of aluminum and graphite in these particles; however, Al could be from matrix or by carbide formation. Deep characterization of these nanoparticles is carried-out at this moment. Additionally, Figure 6 shows a nanofiber found in $\mathrm{Al}_{100}$ composite, lengths of about $800 \mathrm{~nm}$ and diameter lower than $200 \mathrm{~nm}$ were observed. 
Table I.- Identification in samples, composition and experimental conditions

\begin{tabular}{llll}
\hline Identification & Composition & Milled & Extruded \\
\hline $\mathrm{Al}_{\mathrm{p}}$ & Pure Aluminum & --- & $\mathrm{X}$ \\
$\mathrm{Al}_{\mathrm{m}}$ & Pure Aluminum & $\mathrm{X}$ & $\mathrm{X}$ \\
$\mathrm{Al}_{25}$ & $\mathrm{Al}-0.25 \% \mathrm{C}$ & $\mathrm{X}$ & $\mathrm{X}$ \\
$\mathrm{Al}_{50}$ & $\mathrm{Al}-0.50 \% \mathrm{C}$ & $\mathrm{X}$ & $\mathrm{X}$ \\
$\mathrm{Al}_{75}$ & $\mathrm{Al}-0.75 \% \mathrm{C}$ & $\mathrm{X}$ & $\mathrm{X}$ \\
$\mathrm{Al}_{100}$ & $\mathrm{Al}-1.00 \% \mathrm{C}$ & $\mathrm{X}$ & $\mathrm{X}$ \\
\hline
\end{tabular}

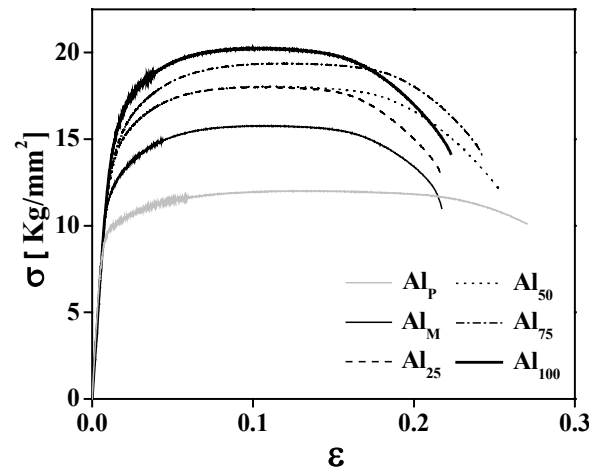

Figure 1.- $\sigma$ vs $\varepsilon$ curves from all samples tested in the extrusion direction.

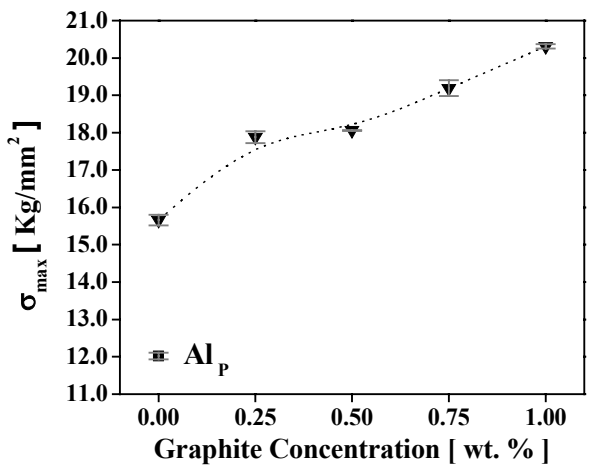

Figure 3.- $\sigma_{\max }$ as a function of graphite contents.

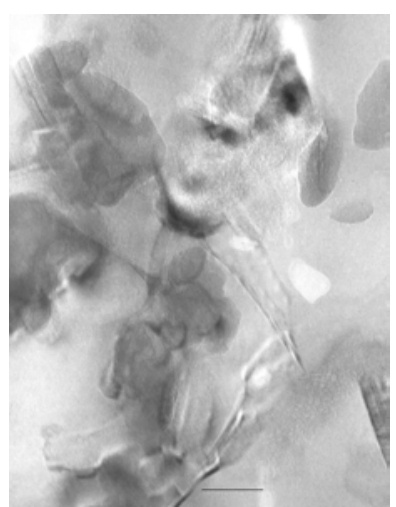

Figure 5.- nanoparticles found in $\mathrm{Al}_{100}$ composites. Particles size is lower than $100 \mathrm{~nm}$.

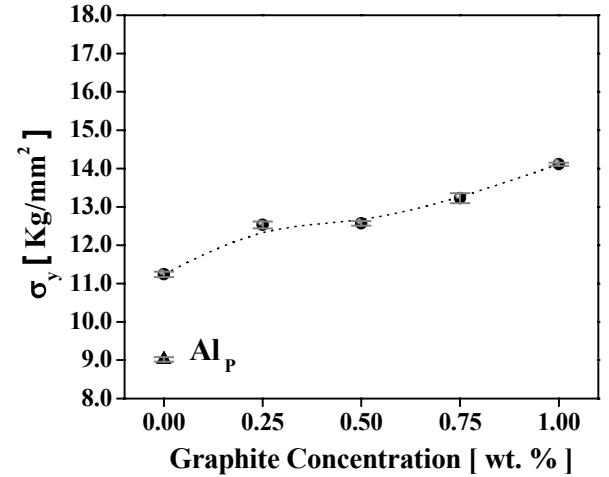

Figure 2.- $\sigma_{\mathrm{y}}$ as a function of graphite contents.

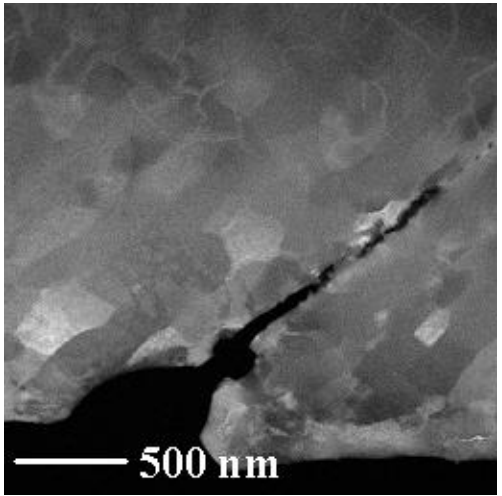

Figure 4.- TEM micrograph from pure aluminum $\left(\mathrm{Al}_{\mathrm{p}}\right.$ sample). Notice the partial recrystallization.

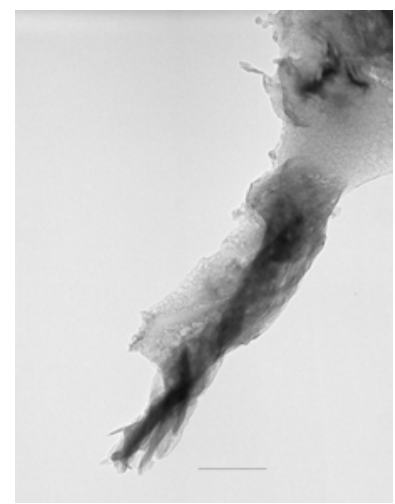

Figure 6.- $\mathrm{Al}-\mathrm{O}-\mathrm{C}$ nanofibers found in $\mathrm{Al}_{100}$ composites. Nanofibers present amorphous structure. 